PROCEEDINGS OF THE

AMERICAN MATHEMATICAL SOCIETY

Volume 125, Number 7, July 1997, Pages 1953-1954

S 0002-9939(97)03916-6

\title{
SPLITTINGS OF CERTAIN FUCHSIAN GROUPS
}

\author{
M. J. DUNWOODY AND M. SAGEEV \\ (Communicated by Ronald M. Solomon)
}

\begin{abstract}
We provide an explicit splitting of Fuchsian groups of type $(1 ; n)$
\end{abstract}
as free products with amalgamation.

Suppose that $G$ is a Fuchsian group of signature $(1 ; n)$, with $n>1$. Algebraically, $G$ can be described by the following presentation: $G=\left\langle a, b \mid[a, b]^{n}\right\rangle$. An old question (see [6]) is whether or not such a group splits as a non-trivial free product with amalgamation. While for many Fuchsian groups it is easy to see geometrically that $G$ has such a splitting, this particular group does not appear to have a splitting which is readily seen geometrically. In the case that $n$ is not a power of 2 , the question was settled by Zieschang [5] and Rosenberger [4] by mapping $G$ onto a Fuchsian group which does have a geometric splitting. Recently, Long, MacLachlan and Reid [3] proved that such a splitting exists for all $n>1$, although an explicit splitting is not provided. In this note, we give an explicit construction of a splitting for all $n>1$. The idea is to take a standard HNN-extension for $G$ and "fold" it in an appropriate way to get the desired splitting.

We give now a brief description of the folding process which achieves the splitting. This discussion is not necessary for the proof of the Theorem, but provides motivation for how one "comes up with it" and may prove useful in other situations in which one wishes to pass from an HNN-extension to a free product with amalgamation. For a general discussion of folding see [2] or [1]. Let $H=\left\langle a, x \mid x^{n}\right\rangle$. It is not hard to see that $G$ splits as an HNN-extension in the following way:

$$
G \cong H *_{\mathbb{Z}}=\left\langle a, x, t \mid x^{n}, a^{t}=a x\right\rangle .
$$

One can get a different HNN-extension as follows. Choose some $z \in H$. Then

$$
G \cong H *_{\mathbb{Z}}=\left\langle a, x, t \mid x^{n}, a^{t}=(a x)^{z}\right\rangle .
$$

(To see this, set $b=z t$ and carry out a Tietze transformation to remove $z$.) This HNN-extension is a graph of groups with one vertex and one edge. One can now subdivide the edge yielding a new graph of groups with two vertices and 2 edges. One can now perform a fold of type IIIA fold as in [2] (see the figure) to obtain a splitting as a free product with amalgamation.

It then remains to choose the $z$ above so that the splitting is non-trivial. There is in fact quite a lot of choice in choosing such a $z$, but we will take $z=x a x$.

We now present the result of this folding process.

Theorem. Suppose $G$ is a group of signature $(1 ; n), n>1$. Let $H, K$, and $L$ be given by the following presentations: $H=\left\langle a, x \mid x^{n}\right\rangle, \quad K=\langle b, w\rangle, \quad L=\langle c, y\rangle$. Let

Received by the editors January 30, 1996.

1991 Mathematics Subject Classification. Primary 20H10.

This work was partially supported by EPSRC Grant GR/K25618. 
$\langle a\rangle$

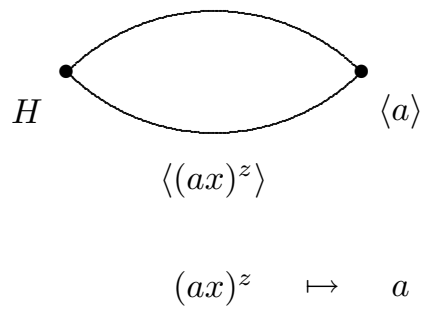

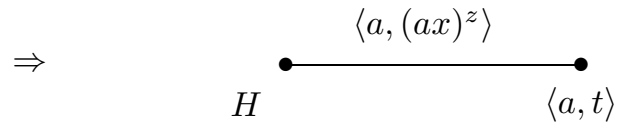

$H \quad\langle a, t\rangle$

$(a x)^{z} \mapsto a^{t}$

The folding operation.

$z=x a x \in H$. Then the following is a non-trivial splitting of $G$ as a free product with amalgamation: $G \cong H *_{L} K$, where the maps $i: L \rightarrow H$ and $j: L \rightarrow K$ are defined by $i(c)=a, i(y)=z^{-1} a x z, j(c)=b$ and $j(y)=w^{-1} b w$.

Proof. First, note that as $K$ is free, the subgroup generated by $b$ and $w^{-1} b w$ is a proper free subgroup of $K$. Thus the map $j$ is a non-surjective injective map. It is also not hard to see that the map $i$ is an injective, non-surjective map. For in any word in $a=i(c)$ and $x^{-1} a^{-1} x^{-1} a x^{2} a x=i(y)$ the exterior $x$ 's of $i(y)$ serve as a barrier for cancellation, so that $i(y)$ and $i(c)$ generate a free subgroup of $H$. (Note that this holds even in the case that $n=2$, in which case $i(y)=x a^{-1} x a^{2} x$.) Thus the group $G^{\prime}=H *_{L} K$ is indeed a non-trivial free product with amalgamation.

It remains to check that $G^{\prime} \cong G$. This involves a few of Tietze tranformations. A presentation for $G^{\prime}$ is

$$
\begin{aligned}
G^{\prime} & =\left\langle a, x, w \mid x^{n}, z^{-1} a x z=w^{-1} a w\right\rangle \\
& =\left\langle a, x, w, b \mid x^{n}, z^{-1} a x z=w^{-1} a w, b=w z^{-1}\right\rangle \\
& =\left\langle a, x, w, b \mid x^{n}, z^{-1} a x z=w^{-1} a w, w=b z\right\rangle \\
& =\left\langle a, x, b \mid x^{n}, z^{-1} a x z=z^{-1} b^{-1} a b z\right\rangle=\left\langle a, x, b \mid x^{n}, x=a^{-1} b^{-1} a b\right\rangle \cong G,
\end{aligned}
$$

as desired.

\section{REFERENCES}

1. M. Bestvina and M. Feighn, Bounding complexity of simplicial group actions on trees, Invent. Math. 103 (1991), 449-469. MR 92c:20044

2. M. J. Dunwoody, Folding Sequences, Preprint, 1994.

3. D. D. Long, C. MacLachlan, and A. W. Reid, Splittings of groups of signature (1;n), Preprint, 1994.

4. G. Rosenberger, Bemerkung zu Einer Arbeit von H. Zeichang, Archiv der Math. 29 (1977), 623-637. MR 57:3259

5. H. Zeichang, Über die Nielsensche Kürzungsmethode in Freien Producten mit Amalgam, Invent. Math. 10 (1970), 4-37.

6. H. Zeichang, Surfaces and Planar Discontinuous Groups, Springer-Verlag, 1980.

Department of Mathematics, University of Southampton, Highfield, Southampton, S017 1BJ, England

E-mail address: mjd@maths.soton.ac.uk

E-mail address: mes@maths.soton.ac.uk 